\title{
Large mode area hybrid multi-trench fiber for anomalous dispersion
}

\author{
Deepak Jain*, Catherine Baskiotis, and Jayanta Kumar Sahu \\ Optoelectronic Research Center, University of Southampton, Southampton SO17 1BJ, UK \\ *dj3g11@orc.soton.ac.uk \\ Abstract: We propose a novel fiber design that shows excellent filtering for higher-order-modes $(>6 \mathrm{~dB} / \mathrm{m})$ and \\ low losses for fundamental mode $(<0.05 \mathrm{~dB} / \mathrm{m})$ at $1064 \mathrm{~nm}$, with an anomalous-dispersion $>72 \mathrm{ps} / \mathrm{nm}-\mathrm{km}$ and effective \\ OCIS codes :(060.2280) Fiber design and manufacturing; (140.3510) Fiber lasers.
} area $>390 \mu \mathrm{m}^{2}$ with good bend robustness.

\section{Introduction}

High power fiber lasers around $1 \mu \mathrm{m}$ have shown great potential owing to the excellent properties of ytterbium ions, especially in continuous wave regime. However, it remains challenging to generate ultrashort pulses due to the normal dispersion of conventional step index fibers around $1 \mu \mathrm{m}$. In order to compensate the dispersion within the laser cavity, fiber with anomalous dispersion can be used as a dispersion compensating element. Several fiber designs with anomalous dispersion around $1 \mu \mathrm{m}$ have been proposed to compensate the dispersion within the laser cavity, such as Photonic Crystal Fibers (PCFs) [1], Hollow Core Photonic Bandgap Fibers (HC-PBGFs) [2], Bragg Fibers [3], and 2D-All Solid Photonic Bandgap Fibers (2D-ASPBGFs) [4]. Recently, a technique of exploiting the anomalous dispersion of Higher Order Mode (HOM) in a few moded fibers has also been demonstrated [5].

PCFs offer high anomalous dispersion ( $76 \mathrm{ps} / \mathrm{nm} . \mathrm{km})$ due to their strong confinement of light in the core but this can be achieved for small Effective Area $\left(\mathrm{A}_{\text {eff }}\right)$ of the Fundamental Mode $(\mathrm{FM})$ which is less than $5 \mu \mathrm{m}^{2}[1]$. Anomalous dispersion has also been demonstrated in Bragg fibers [3]. For example, recently a Bragg fiber with mode field diameter (MFD) 7 $\mu \mathrm{m}$ at $1.064 \mu \mathrm{m}$ with dispersion of $13 \mathrm{ps} /(\mathrm{nm} . \mathrm{km})$ and low losses of the FM $(0.06$ $\mathrm{dB} / \mathrm{m}$ ) has been demonstrated. However, both of these solutions provide low threshold of non-linear effects due to their limited $\mathrm{A}_{\text {eff. }}$. Although, this problem of low threshold of non-linear effects can be solved by using HC-PBGFs, which provide anomalous dispersion around $1 \mu \mathrm{m}$ but they are difficult to splice with standard solid core fibers [2]. Few moded fiber can provide anomalous dispersion at large $\mathrm{A}_{\text {eff }}$ of the $\mathrm{FM}$, but this technique requires long period gratings, which make the system cumbersome [5]. 2D-ASPBGFs show high anomalous dispersion at the long wavelength edge of Photonic Bandgap (PBG), for example a 2D-ASPBGFs, with FM losses $\sim 0.07 \mathrm{~dB} / \mathrm{m}$ and MFD $\sim 9 \mu \mathrm{m}$ at $1.04 \mu \mathrm{m}$, can achieve a dispersion of $90 \mathrm{ps} /(\mathrm{nm} . \mathrm{km})$ [4]. It is worth noting that anomalous dispersion in 2D-ASPBGFs is obtained at the long wavelength edge of the PBG which leads to high losses for the FM. The losses of the FM mode start increasing once the $\mathrm{A}_{\text {eff }}$ of the FM increases and it becomes difficult to obtain low losses for the FM at large $\mathrm{A}_{\text {eff }}$ in the anomalous dispersion region. Hybrid Photonic Crystal Fiber (H-PCF), in which a line of air holes is replaced by the high index rods, presents PBG nature in PCF, thanks to the resonant action of the highindex rods [7-8]. A similar concept has also been applied to the step index fibers [9]. These hybrid fibers can achieve anomalous dispersion at the long wavelength edge of bandgap due to the PBG action of the high index rods, while maintaining low losses due to presence of less number of rods compared to 2D-ASPBGFs. However, these fibers are limited to mode area scaling. Moreover, the splicing of PCFs still remains a challenge. Recently, we proposed Hybrid Multi-trench Fibers (H-MTF) for mode area scaling, which are all-solid structures, presenting a cylindrical symmetry $[10,11]$. They ensure the filtering of the HOMs by couplings to the resonant rings of their cladding, while the high index rods along one axis of the fiber provide similar characteristics as 2D-ASPBGFs. In this paper, we investigate the dispersion properties of H-MTF.

\section{Hybrid Multi Trench Fiber (H-MTF)}

Fig. 1(a) presents the H-MTF with the high-index rods (Ge-doped silica) positioned along the X-axis of the MTF. Fig. 1(b) shows the refractive index profile of the fiber along $X$ and $Y$ axis. The parameters of the MTF are: core $\operatorname{radius}\left(\mathrm{r}_{\mathrm{c}}\right)=15 \mu \mathrm{m}$, trench thickness $(\mathrm{t})=2.0 \mu \mathrm{m}$, trench refractive-index w.r.t core index $(\Delta \mathrm{n}$. $)=-0.006$, and resonantring thickness $(\mathrm{d})=8.5 \mu \mathrm{m}$. The high-index rods have a step-index profile with core $\operatorname{radius}\left(\mathrm{r}_{\mathrm{ch}}\right)=3.0 \mu \mathrm{m}$, outer cladding radius $\left(\mathrm{r}_{\mathrm{oh}}\right)=4.0 \mu \mathrm{m}$, and refractive-index w.r.t pure-silica cladding $\left(\Delta \mathrm{n}_{+}\right)=0.035$. Numerical simulations have been carried out using the Finite Element Method (FEM). Fig. 1(c) shows the computed bending losses of the FM and the HOM having the lowest losses level, at a wavelength of $1064 \mathrm{~nm}$, of the MTF and H- MTF, for two different bend orientations. A single mode operation can be ensured in the $14 \mathrm{~cm}$ to $20 \mathrm{~cm}$ bend radius range, with FM bend losses being lower than $0.051 \mathrm{~dB} / \mathrm{m}$ and all other HOMs losses, being larger than $5 \mathrm{~dB} / \mathrm{m}$. The $A_{\text {eff }}$ remains larger than $390 \mu \mathrm{m}^{2}$ for this bend radius range (including the bend-induced $\mathrm{A}_{\mathrm{eff}}$ reduction). 


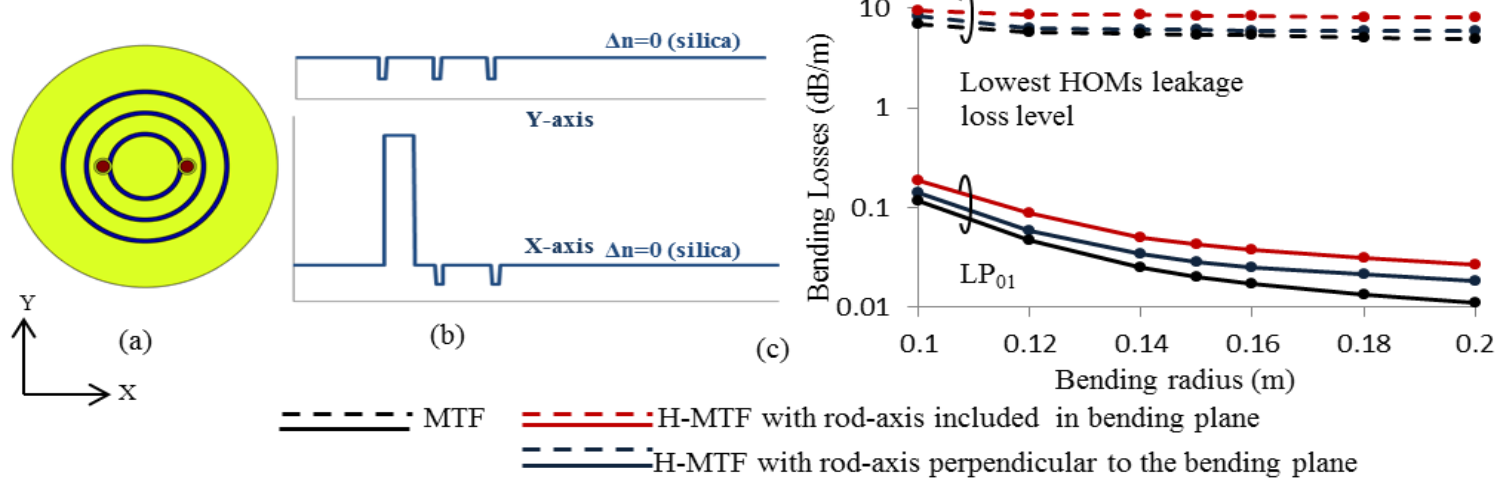

Fig. 1(a) Schematic cross-section of H-MTF. Blue, yellow-green, and brown colours represent F-doped, undoped, and Gedoped silica regions respectively. (b) Refractive index profile of H-MTF along the X and Y axis (c) Computed bending losses of the MTF and H-MTF for two orthogonal bend orientations.

\section{Anomalous Dispersion}

Fig. 2(a), 2(b) and 2(c) show the calculated wavelength spectrums for the leakage losses, the dispersion, and the power fraction in the core respectively, of the FM for both MTF and H-MTF in unbent case, for the fiber parameters used in Fig. 1. Fig. 2(c) also shows the normalized electric field of the FM at certain wavelengths. Additional high index rods in the MTF provide an additional guidance mechanism, which is clear from the spectrums of the H-MTF. H-MTF supports transmission of light in the core between the cut-off wavelengths of the high index rods' modes. Fig. 2(c) shows the filtering of the power from the core in the $835-865 \mathrm{~nm}, 935-945 \mathrm{~nm}, 1075-1090 \mathrm{~nm}$, and $1165-$ $1175 \mathrm{~nm}$ wavelength regions, which correspond to the cut-off wavelengths of the $\mathrm{LP}_{03}-\mathrm{LP}_{22}, \mathrm{LP}_{41}, \mathrm{LP}_{12}$, and $\mathrm{LP}_{31}$ modes of the high index rods respectively. Fig. 2(b) shows the dispersion spectrum of MTF and H-MTF. In case of H-MTF, between the cut off wavelengths of two modes of high index rods, it shows normal dispersion $(\mathrm{D}<0)$ at short wavelength edge and anomalous dispersion $(D>0)$ at long wavelength edge with a point of inflection $(D=0)$ between them (which is similar to 2D-ASPBGFs). It is worth noting that the obtained spectrums for H-MTF are similar to that of a 2D-ASPBGF, although in the present case, the transmission region of the rods (forbidden region of the core) is quite narrow as compared to 2D-ASPBGFs. It is well known that in a 2D-ASPBGFs, the wavelength region over which anti-crossing between FM of core and high index rods' mode take place is broad due to formation of broadband supermode of high index rods' modes [13]. This broadband supermode formation took place due to mutual coupling between high index rods' modes. In the present case of H-MTF, limited number of high index rods avoids the formation of broadband supermode, which results in narrow forbidden region of core [9, 11].
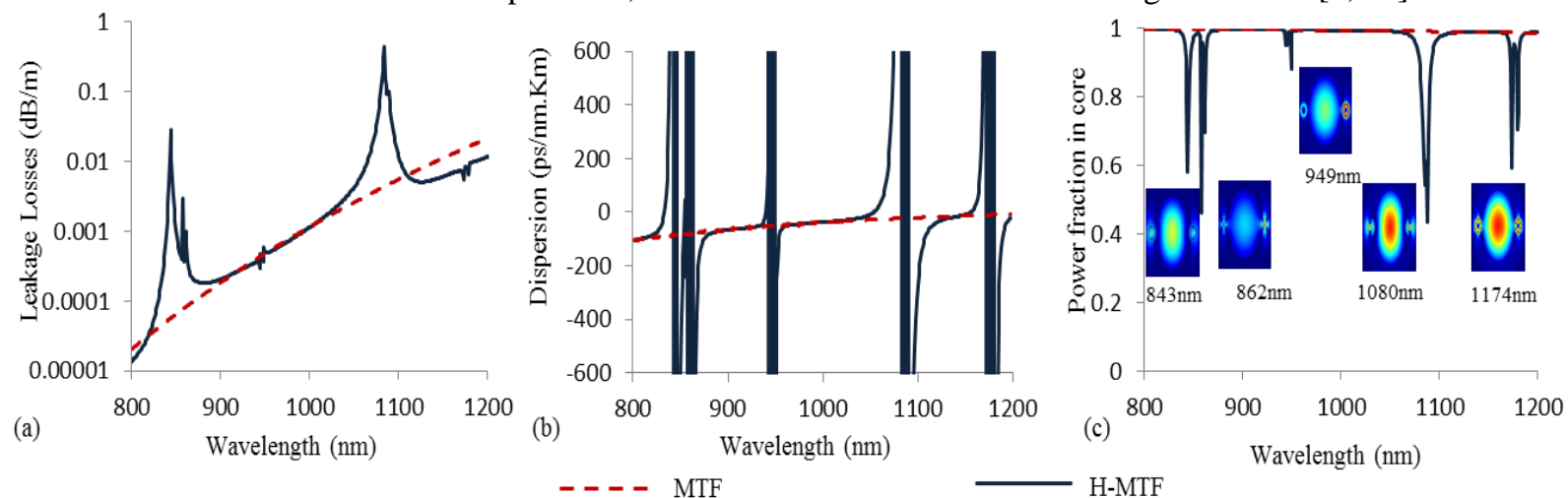

Fig. 2(a), 2(b), and 2(c) shows calculated leakage losses, dispersion, and power fraction of the FM of MTF and H-MTF respectively in the case of an unbent fibre of Fig. 1(c). Inset also shows normalized electric field of the fundamental core mode of H-MTF at certain wavelengths.

Fig. 3 shows the bend loss and power fraction wavelength spectrums of the FM, at a bend radius of $15 \mathrm{~cm}$, for two different cases (namely rods axis included in the bending plane and orthogonal to it). We obtained an anomalous dispersion larger than $72 \mathrm{ps} / \mathrm{nm} . \mathrm{km}$ at wavelength of $1064 \mathrm{~nm}$ with $\mathrm{A}_{\text {eff }}$ larger than $390 \mu \mathrm{m}^{2}$. The losses of the FM remain lower than $0.043 \mathrm{~dB} / \mathrm{m}$, while ensuring high losses to the HOMs ( $>6 \mathrm{~dB} / \mathrm{m})$. Moreover, by tuning the parameters (refractive index and core diameter) of the high-index rods, the zero dispersion wavelength (ZDW) can 
be shifted to other wavelengths in order to achieve anomalous dispersion at a desired wavelength. It is worth noting that in conventional 2D-ASPBGFs, a large anomalous dispersion at the long wavelength edge of a bandgap can be obtained but at the cost of large leakage losses of the FM [6]. Therefore, it is an interesting finding of our study that a large anomalous dispersion can be achieved at the long wavelength edge of the bandgap, which is similar to 2DASPBGFs. However, unlike 2D-ASPBGFs, this is achieved while ensuring low losses of the FM for a large $\mathrm{A}_{\text {eff. }}$ To the best of our knowledge, this is the first report showing the feasibility of achieving large mode area single-mode operation, with large anomalous dispersion in an all-solid fiber around $1 \mu \mathrm{m}$ regime, with easy fabrication.

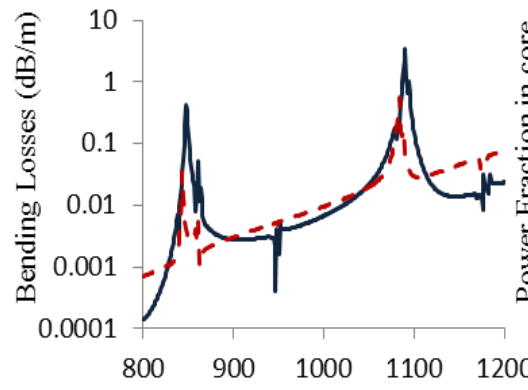

(a) Wavelength $(\mathrm{nm})$

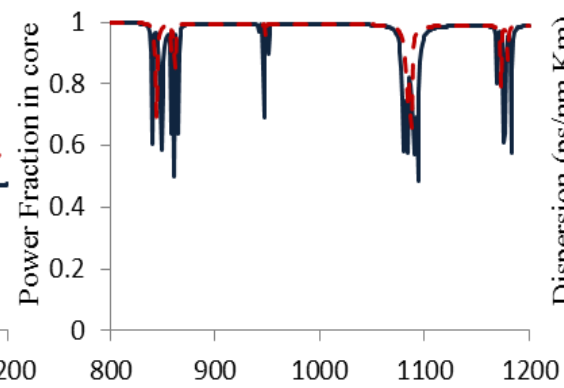

(b)

Wavelength (nm)

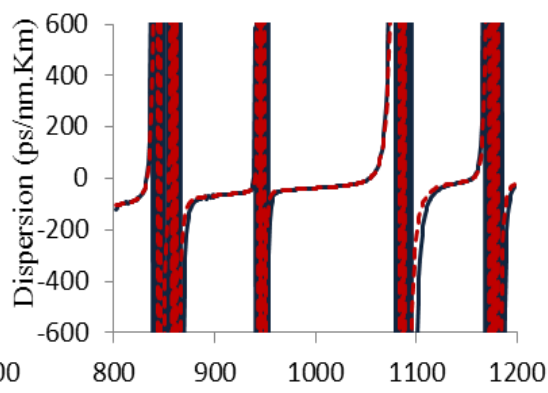

(C)

Wavelength (nm)

Rods-axis included in the bending plane

- - - - Rods-axis included perpendicular to the bending plane

Fig. 3 Bend loss spectrum (a), power fraction spectrum (b), dispersion (c) of the fundamental core mode of the H-MTF of the Fig. 1(c) at a $15 \mathrm{~cm}$ bend radius, for two orthogonal bending orientations.

\section{Conclusion}

We have studied the feasibility of employing two guiding mechanisms, namely total internal reflection and antiresonance, simultaneously in a MTF by using additional high index rods. The overall properties of the hybrid fiber, including dispersion spectrum, losses and power fraction in core, have similar nature as that of conventional 2DSPBGFs, due to anti-crossings between the FM core mode and high index rods' modes. On the other hand, single mode propagation of core is ensured by the resonant coupling between the HOMs of core and resonant rings' modes of MTF. Using this design, we have simultaneously obtained a loss-loss single mode operation, large effective area for the FM, and large anomalous dispersion with good bend robustness.

\section{References}

[1] H. Lim et. al., "Femtosecond ytterbium fiber laser with photonic crystal fiber for dispersion control," Opt. Express 10, 1497-1502 (2002).

[2] H. Lim et. al., "Control of dispersion in a femtosecond ytterbium laser by use of hollow-core photonic bandgap fiber," Opt. Exp. 12, 22312235 (2004).

[3] S. S. Aleshkina et. al., "Low-loss hybrid fiber with zero dispersion wavelength shifted to $1 \mu$ m," Opt. Exp., 21, 23838-23843 (2013).

[4] A. Isomaki et. al., "All-fiber ytterbium soliton mode-locked laser with dispersion control by solid-core photonic bandgap fiber", Opt. Exp. 14, 4368-4373 (2006).

[5] S. Ramachandaran et. al. , “Anomalous dispersion in a solid, silica-based fiber,” Opt. Letter 31, 2532-2534 (2006).

[6] Q. Fang et. al., "Dispersion design of all-solid photonic bandgap fiber," JOSA B, 24, 2899-2905, (2007).

[7] A. Cerqueira et. al., "Hybrid photonic crystal fibre," Opt. Exp. 14, 926-931, (2006).

[8] S. R. Petersen et. al., "Degenerate four wave mixing in large mode area hybrid photonic crystal fibers," Opt. Exp. 21, 18111-18124 (2013).

[9] R. Goto et. al., "Birefringent all-solid hybrid microstructured fiber," Opt. Exp. 16, 18752-18763 (2008).

[10] D. Jain et. al., "Mode area scaling with Multi-trench rod-type fibers," Opt. Exp. 21, 1448-1455 (2013).

[11] D. Jain et. al., "Large mode area hybrid multi-trench fiber for spectral filtering," presented at FIO, Florida, USA FW2A.3 (Oct. 2013 ).

[12] A. W. Synder et. al., "Optical waveguide theory," (Chapman and Hall, New York, 1983).

[13] J. Laegsgaard et. al., "Gap formation and guided modes in photonic bandgap fibers with high-index rods," J. Opt. A: P. App. Opt.6 (2004).

\section{Acknowledgement}

The work is supported by the EPSRC Centre for the Innovative manufacturing in Photonics EP/HO2607X/1. 